\title{
A theoretical study of solvent effects on the characteristics of the intramolecular hydrogen bond in Droxidopa
}

\author{
MEHDI YOOSEFIAN, HASSAN KARIMI-MALEH and AFSANEH L SANATI* \\ Department of Chemistry, Graduate University of Advanced Technology, Kerman, Iran \\ e-mail: 1.sanatia@yahoo.com
}

MS received 04 May 2014; revised 27 January 2015; accepted 28 January 2015

\begin{abstract}
The molecular structures and intramolecular hydrogen bond of Droxidopa have been investigated with density functional theory. It is found that strong hydrogen bonds $(\mathrm{O}-\mathrm{H} \ldots \mathrm{N}$ and $\mathrm{O} \ldots \mathrm{H}-\mathrm{O})$ exist in the title compound. These hydrogen bonds play essential roles in determining conformational preferences and energy, which would have important effects in biological activity mechanisms that will strongly influence its characteristics in solution. A computational study of a representative number of actual and model structures was carried out in five solvents with different polarities and different types of interactions with solute molecules: water, ethanol, carbon tetrachloride, dimethyl sulfoxide, and tetrahydrofuran, utilizing the polarizable continuum model (PCM) model. The calculations were performed at the B3LYP/6-311++G(d,p) level of theory. In addition, the topological properties of the electron density distributions for $\mathrm{O}-\mathrm{H} . . \mathrm{N}(\mathrm{O})$ intramolecular hydrogen bond were analyzed in terms of the Bader's theory of atoms in molecules. Furthermore, the analyses of different hydrogen bonds in this molecule by quantum theory of natural bond orbital (NBO) methods support the density functional theory (DFT) results.
\end{abstract}

Keywords. Droxidopa; intramolecular hydrogen bond; solvent effect; AIM analysis; NBO.

\section{Introduction}

The importance of hydrogen bond cannot be overstated. It is strong enough to influence the arrangement of molecules in crystals and even the geometry of molecules participating in these interactions. ${ }^{1-8}$ They influence the physicochemical properties ${ }^{9-13}$ and play relevant roles in important aspects of biological activity mechanisms like selective binding, molecular recognition and in specific activities (e.g., anti-tumour activity. ${ }^{14,15}$ The presence of a solvent affects molecular properties such as bond length, electron population on atoms, energy level separations, ${ }^{16,17}$ relative stabilities of different conformers of a molecule or different isomers, ${ }^{18,19}$ polarizabilities, dipole moments, NMR and ESR parameters. ${ }^{20,21}$ Its influence on important aspects of the mechanisms of biological activities, such as molecular recognition ${ }^{22}$ or interactions with the receptor, ${ }^{23}$ and the fact that biological activities occur in some medium within living organisms ${ }^{24}$ provide strong motivation for the study of the solvent effects on the characteristics of biologically active molecules. The current study investigates the influence of the presence of a medium (solvent) on

*For correspondence the characteristics of the intramolecular hydrogen bond (IHB) typical of Droxidopa (figure 1). The study in solution aims at verifying whether and to what extent those patterns remain valid, or new patterns emerge, under different types of solute-solvent interactions.

Droxidopa is an international nonproprietary name (INN) for a synthetic amino acid precursor of norepinephrine, whose systematic (IUPAC) name is $(2 \mathrm{R}, 3 \mathrm{~S})$ 2-amino-3-(3,4-dihydroxyphenyl)-3-hydroxypropanoic acid (L-DOPS). Droxidopa is a psychoactive drug and a synthetic amino acid precursor that acts as a prodrug to the neurotransmitters norepinephrine (noradrenaline) and epinephrine (adrenaline). ${ }^{25}$ Unlike norepinephrine and epinephrine themselves, L-DOPS is capable of crossing the protective blood-brain barrier (BBB). ${ }^{25}$ Data from clinical studies and post-marketing surveillance programs conducted in Japan show that the most commonly reported adverse drug reactions with Droxidopa are increased blood pressure, nausea, and headache. In clinical studies to date, data suggest that Droxidopa is well tolerated and effective as a norepinephrine precursor. ${ }^{26}$

Five solvents exerting different types of interactions with the solute molecules are selected: water and ethanol (which are capable of forming intermolecular Hbonds with the solute, with both donor and acceptor 


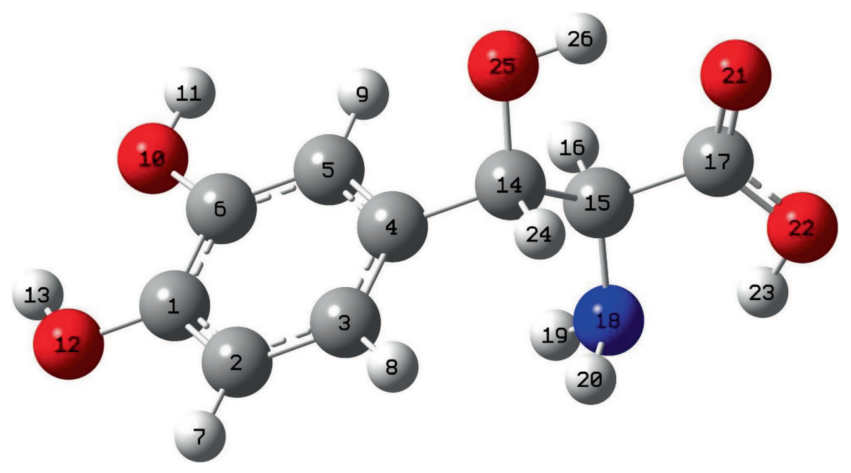

Figure 1. The geometry of Droxidopa and the numbering of the atoms.

roles, and also among themselves), tetrahydrofuran and dimethyl sulfoxide (which can only form intermolecular H-bonds with the solute, with acceptor role), and carbon tetrachloride (which cannot form H-bonds). Their different dielectric constants ( 78.4 for water, 46.7 for dimethyl sulfoxide, 24.6 for ethanol, 7.6 for tetrahydrofuran, 2.3 for carbon tetrachloride) enable informative comparisons from the results of continuum model $\left(\mathrm{PCM}^{27,28}\right)$ calculations. Their different polarities adequately cover the polarity range of possible environments in which Droxidopa molecules may be preferably present within a living organism, according to preference of individual compounds for more or less polar solvents.

\section{Computational Details}

All DFT calculations were performed with the Gaussian $03^{29}$ with the default convergence criteria without any constraint on the geometry. The geometry optimizations were carried out using the B3LYP method with 6-311++ $\mathrm{G}^{* *}$ basis set. Calculations in solution were performed with the polarizable continuum model (PCM) as employed in the Gaussian 03 package, with its default settings for building the cavity around the solute, with simple United Atom Topological Model (UAO) for the atomic radii (default values) and average tesserae area $0.200 \AA .^{2}$ Calculations used the vacuum optimized geometries as inputs, and performed reoptimization in solution at the same level of theory (reoptimization being the only choice for the study of geometry-related characteristics similar to the parameters of the IHB, besides its general importance for the quality of the description of the solvation phenomenon). The nature of the intramolecular hydrogen bonds existing within Droxidopa in vacuum and in the five solvents was studied by means of the Bader theory of atoms in molecules (AIM). The calculated electron density, $\rho$, and its second derivative, $\nabla^{2} \rho$, were used for describing the nature of the intramolecular $\mathrm{O}-\mathrm{H} . . .(\mathrm{O}) \mathrm{N}$ bonds. The AIM2000 program was used to find the bond critical points (BCPs) and analyze. ${ }^{30}$ Finally, for comprehensive understanding of the nature of intramolecular interactions, the natural bond orbital (NBO) method was utilized. ${ }^{31}$ For the sake of conciseness, the environment will be denoted with the following acronyms on reporting values: VAC (vacuum), CC (carbon tetrachloride), THF (tetrahydrofuran), DMSO (dimethyl sulfoxide), ET (ethanol), and AQ (water).

\section{Results and Discussion}

\subsection{Molecular geometry}

The geometry of Droxidopa and the numbering of atoms and the geometrical parameters of selected parameters in VAC and in the five solvents are given in figure 1 and table 1 , respectively.

The same atom numbering as for the study in VAC is utilized for all the environments to facilitate the comparisons. The geometrical parameters showed that the $\mathrm{N} . . . \mathrm{H}, \mathrm{O} \ldots \mathrm{H}$ distances and $\theta_{(\mathrm{O}-\mathrm{H} \ldots \mathrm{N})}, \theta_{(\mathrm{O}-\mathrm{H} \ldots \mathrm{O})}$ in Droxidopa molecule are in the range of 1.834-1.904, 1.929$1.938-\AA$ and $124.57-126.96,139.57-141.72$ degree, respectively. It is well-known that the geometrical parameters of the HB reflect the strength of the bond. In many cases, there is a clear relationship between these parameters and the strength of hydrogen bond. The smaller the N...H and O...H distances and the larger $\theta_{(\mathrm{O}-\mathrm{H} \ldots \mathrm{N})}$ and $\theta_{(\mathrm{O}-\mathrm{H} \ldots \mathrm{O})}$ angle in the Droxidopa in vacuum and in the five solvents, the stronger is intramolecular hydrogen bonds. Results in table 1 show that in AQ solution, the $\theta_{(\mathrm{O}-\mathrm{H} \ldots \mathrm{N})}$ and $\theta_{(\mathrm{O}-\mathrm{H} \ldots \mathrm{O})}$ angles are larger and both $\mathrm{N}$...H and $\mathrm{O} . . . \mathrm{H}$ intramolecular hydrogen bonds are stronger than the other solutions. These parameters in CC solution are closed to VAC environment, because the dielectric constant of $\mathrm{CC}$ is very small. As shown in this table, analysis of bond lengths indicates that $\mathrm{r}_{\mathrm{C}-\mathrm{O}}$ are lengthened, with respect to the Droxidopa molecule in VAC. These lengthening of single bonds could be attributed to the increasing quantification of electron delocalization, which in turn causes the hydrogen bond to become stronger than that for Droxidopa molecule in VAC. Therefore, among the environments studied in this work, the lengthening of $\mathrm{C}-\mathrm{O}$ bonds is observed in solution. Alternatively, the main solvent effect is shortening the $\mathrm{N} . . . \mathrm{H}$ and $\mathrm{O}$...H distances compared with the corresponding value in VAC. These structural changes in Droxidopa suggest a stronger hydrogen bond in solutions than that in VAC. 


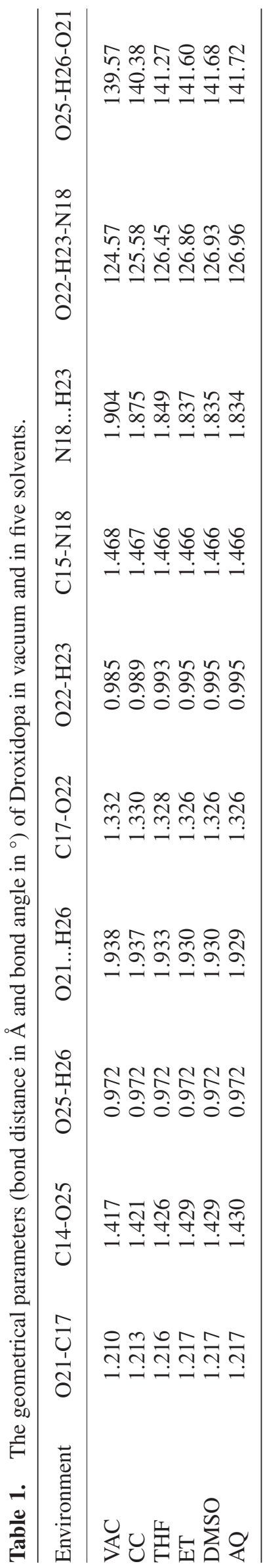

\section{$3.2 \quad H$-bond energies}

One of the most important features of intramolecular H-bond is its strength. The IHB energy plays a significant role in conformational preference, in which its value mainly depends on the choice of the resonance state. Many authors have devised various methods to estimate the energy of IHB. In the Schuster method, ${ }^{32}$ it is assumed that the energy difference between the close (with $\mathrm{HB}$ ) and open (without HB) conformers is equal to $\mathrm{HB}$ energy. But this energy gap also contains some conformational contributions that cannot be a direct measure of the HB energy. In recent years, the powerful method of Espinosa ${ }^{33}$ is often applied for the estimation of H-bond energy. In this method, the $\mathrm{H}$ bond energies may be estimated from the properties of bond critical points. Espinosa and co-workers have proposed a simple relationship between the hydrogen bond energy and the local electron potential energy density $\mathrm{V}\left(\mathrm{r}_{\mathrm{CP}}\right)$ at $\rho_{\mathrm{O}(\mathrm{N}) \ldots \mathrm{H}}$ :

$$
\mathrm{E}_{\mathrm{HB}}=1 / 2 \mathrm{~V}\left(\mathrm{r}_{\mathrm{CP}}\right)
$$

Corresponding to the Abramov's relation ${ }^{34}$ at the critical point:

$$
G\left(r_{C P}\right)=\left(\frac{3}{10}\right)\left(3 \pi^{2}\right)^{\frac{2}{3}} \rho_{\left(r_{C P}\right)}^{\frac{5}{3}}+\left(\frac{1}{6}\right) \nabla^{2} \rho\left(r_{C P}\right)
$$

The local potential energy density $\mathrm{V}\left(\mathrm{r}_{\mathrm{CP}}\right)$ can be obtained from the virial equation:

$$
2 G\left(r_{C P}\right)+V\left(r_{C P}\right)=\left(\frac{1}{4}\right) \nabla^{2} \rho\left(r_{C P}\right)
$$

All values in the relations mentioned above are expressed in atomic units. $\mathrm{E}_{\mathrm{HB}}$ energies calculated from the potential energy densities of O...H and N...H contacts are included in table 2 . These values suggest that the H-bond strength in solutions is relatively stronger than VAC, which is in agreement with calculated geometrical parameters (table 1).

It is worth mentioning that $\mathrm{E}_{\mathrm{HB}}$ correlates to the geometrical parameters, which are usually assumed to be good descriptors of the H-bond strength. The linear correlation coefficient for the dependency of $E_{\mathrm{HB}}$ versus $\mathrm{r}_{\mathrm{O} \ldots \mathrm{H}}$ and $\mathrm{r}_{\mathrm{N} \ldots \mathrm{H}}$ is 0.989 and 0.998 , respectively. It means that $\mathrm{E}_{\mathrm{HB}}$ is a good description of the $\mathrm{H}$-bond strength. Furthermore, as shown in figure 2 , there are very excellent logarithmic correlation coefficients for the dependency of $\mathrm{E}_{\mathrm{HB}}$ versus dielectric constant $(\varepsilon)$ as the following equations for both $\mathrm{N}$...H and $\mathrm{O}$...H hydrogen bond strength:

$$
\begin{aligned}
& E_{H B}=-0.16 \ln \varepsilon-29.86 \text { for O-H...O IHB and } \\
& E_{H B}=-1.63 \ln \varepsilon-45.62 \text { for O-H...N IHB }
\end{aligned}
$$



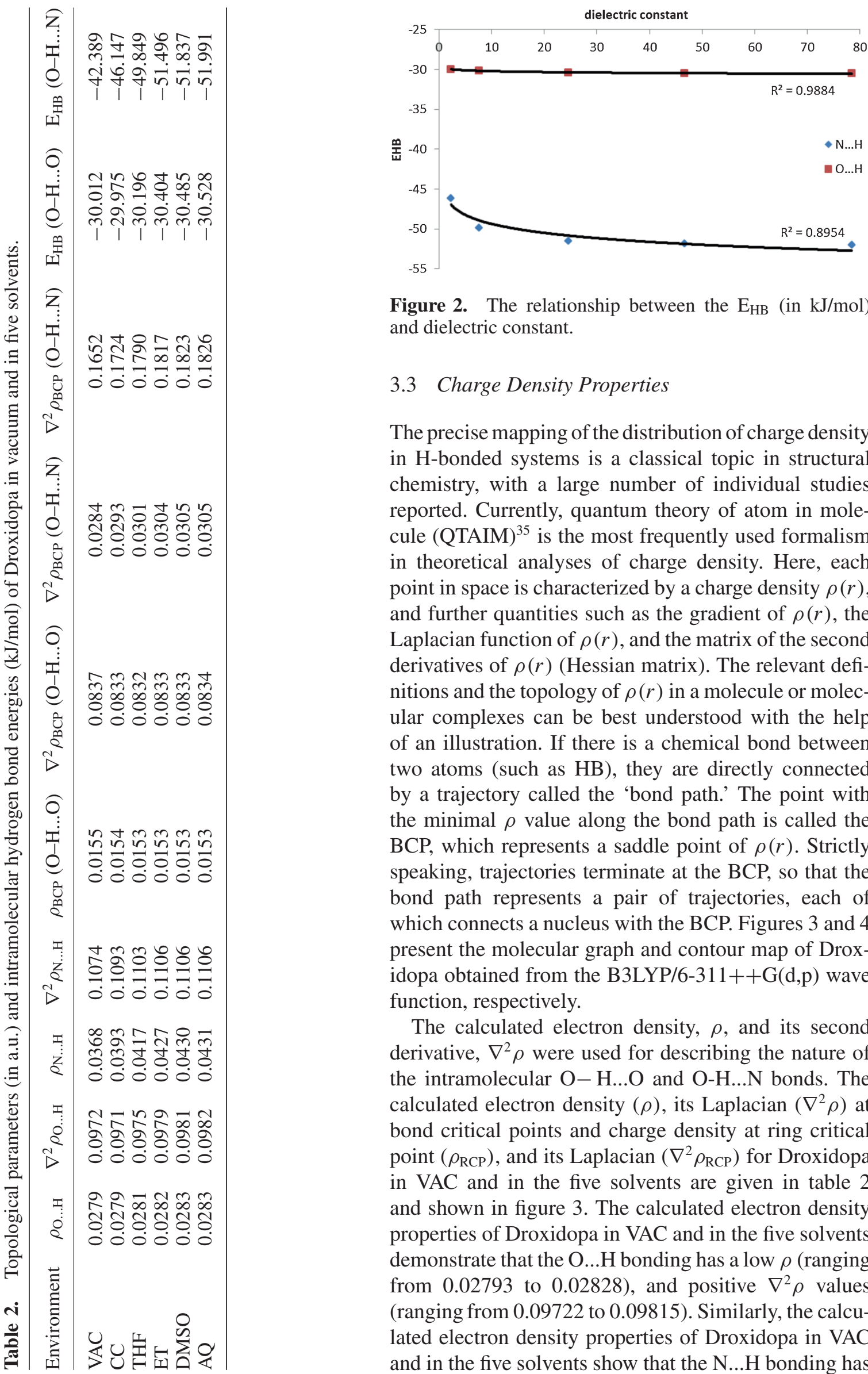

Figure 2. The relationship between the $\mathrm{E}_{\mathrm{HB}}$ (in $\mathrm{kJ} / \mathrm{mol}$ ) and dielectric constant.

\subsection{Charge Density Properties}

The precise mapping of the distribution of charge density in H-bonded systems is a classical topic in structural chemistry, with a large number of individual studies reported. Currently, quantum theory of atom in molecule (QTAIM) ${ }^{35}$ is the most frequently used formalism in theoretical analyses of charge density. Here, each point in space is characterized by a charge density $\rho(r)$, and further quantities such as the gradient of $\rho(r)$, the Laplacian function of $\rho(r)$, and the matrix of the second derivatives of $\rho(r)$ (Hessian matrix). The relevant definitions and the topology of $\rho(r)$ in a molecule or molecular complexes can be best understood with the help of an illustration. If there is a chemical bond between two atoms (such as HB), they are directly connected by a trajectory called the 'bond path.' The point with the minimal $\rho$ value along the bond path is called the $\mathrm{BCP}$, which represents a saddle point of $\rho(r)$. Strictly speaking, trajectories terminate at the $\mathrm{BCP}$, so that the bond path represents a pair of trajectories, each of which connects a nucleus with the BCP. Figures 3 and 4 present the molecular graph and contour map of Droxidopa obtained from the B3LYP/6-311++G(d,p) wave function, respectively.

The calculated electron density, $\rho$, and its second derivative, $\nabla^{2} \rho$ were used for describing the nature of the intramolecular $\mathrm{O}-\mathrm{H} . . . \mathrm{O}$ and $\mathrm{O}-\mathrm{H} . . . \mathrm{N}$ bonds. The calculated electron density $(\rho)$, its Laplacian $\left(\nabla^{2} \rho\right)$ at bond critical points and charge density at ring critical point $\left(\rho_{\mathrm{RCP}}\right)$, and its Laplacian $\left(\nabla^{2} \rho_{\mathrm{RCP}}\right)$ for Droxidopa in VAC and in the five solvents are given in table 2 and shown in figure 3. The calculated electron density properties of Droxidopa in VAC and in the five solvents demonstrate that the $\mathrm{O}$...H bonding has a low $\rho$ (ranging from 0.02793 to 0.02828 ), and positive $\nabla^{2} \rho$ values (ranging from 0.09722 to 0.09815). Similarly, the calculated electron density properties of Droxidopa in VAC and in the five solvents show that the N...H bonding has 


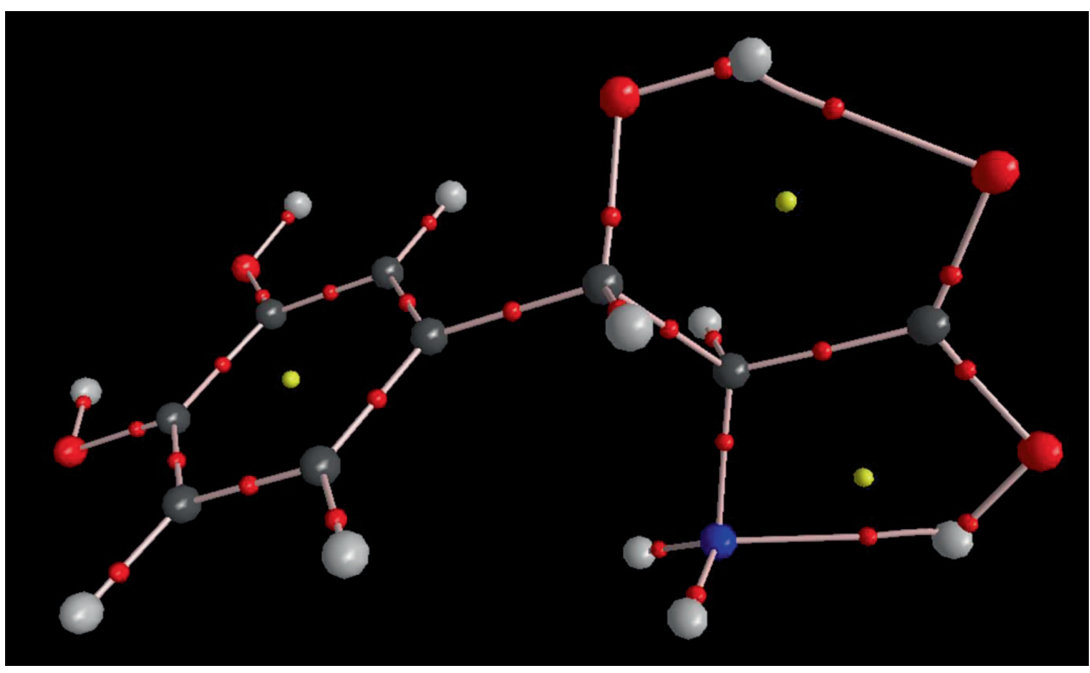

Figure 3. The molecular graphs of Droxidopa obtained from the B3LYP/6-311++G(d,p) wave function.

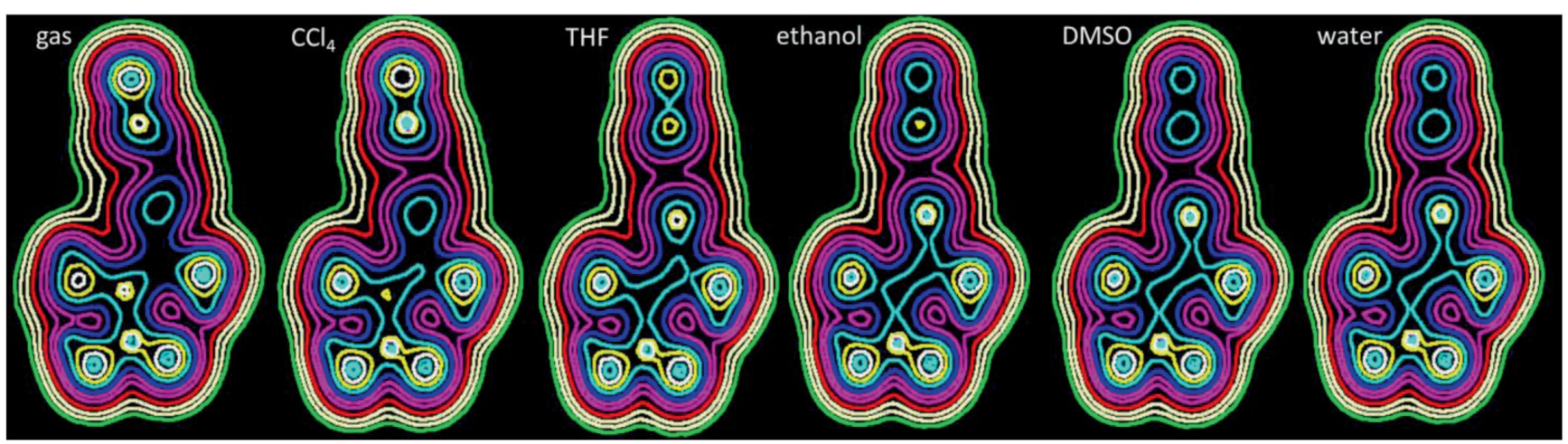

Figure 4. The contour map of Droxidopa in vacuum and in five solvents obtained from the B3LYP/6-311++G(d,p) wave function in plane with three selected points: $\mathrm{A}(2.42,3.06,2.07), \mathrm{B}(7.33,1.88,0.82)$, and $\mathrm{C}(7.97,-1.56,-1.52)$.

low $\rho$ (ranging from 0.03676 to 0.04307 ), and positive $\nabla^{2} \rho$ values (ranging from 0.10743 to 0.1063 ). These properties are typical for closed-shell interactions as HBs and indicate electrostatic character of the O...H and $\mathrm{N}$...H bonding. With considering calculated topological parameters, we can conclude that the main solvent effect is an increase in electronic densities at the ring critical points $\left(\rho_{\mathrm{RCP}}\right)$. This fact indicates that the hydrogen-bond strength in solution is stronger than that of the VAC. Another topic analyzed here is the existence of the ring critical point (RCP) for closed configurations. The $\nabla^{2} \rho_{\mathrm{RCP}}$ is a point of the minimum electron density within the ring surface and a maximum on the ring line. ${ }^{36}$

As shown in figure 4, the density value is arbitrarily terminated at the positions of the carbon, oxygen, and nitrogen nuclei. The lower maps illustrate the trajectories traced out by $\rho$, the gradient vectors of the density. The contour map is overlaid with the trajectories of $\rho$ associated with the bond cps that define the bond paths and the intersection of the interatomic surfaces with the plane of the diagram.

It has been mentioned recently that for IHB, there is a correlation between the electron density at the bond critical point corresponding to the contact within the $\mathrm{H}$-bridge and the electron density at the RCP. ${ }^{11}$ There is a linear relationship between $E_{\mathrm{HB}}$ and $\nabla^{2} \rho$ at the RCP. The corresponding correlation coefficient amounts to 0.998 for N...H bond (with equation as $\left.E_{\mathrm{HB}}=-554.1 \nabla^{2} \rho_{\mathrm{RCP}}+49.234\right)$. This implies that the properties of the RCP values could be very useful to estimate the strength of IHB. Their values permit us to have a better understanding of these novel correlations. The derived relationships from these graphs empower us to acquire other physically meaningful results.

\section{$3.4 \quad N B O$ analysis}

The natural bond orbital (NBO) analyses were applied to evaluate the hydrogen bond strength in the Droxidopa 
in VAC and in the five solvents. The NBO method shows that for typical hydrogen bonding, a two-electron $n_{B} \rightarrow \sigma_{X H}^{*}$ intermolecular donor-acceptor interaction exists where electron density from the lone pair $n_{B}$ of the $\mathrm{H}$-acceptor delocalizes into the unfilled $\sigma_{X H}^{*}$ antibonding orbital of the H-donor. The $n_{B} \rightarrow \sigma_{X H}^{*}$ orbital overlap is characteristic for hydrogen bonding interaction. The hydrogen bond formation leads to an increase in the occupancy of the $\sigma_{X H}^{*}$ antibond orbital and hence the weakening and lengthening of the $\mathrm{X}-\mathrm{H}$ bond. This leads to the red-shifted $v_{\mathrm{X}-\mathrm{H}}$ stretching frequency. Therefore, electron delocalization or charge transfer (CT) effects between $n_{B}$ and $\sigma_{X H}^{*}$ may be estimated by second-order perturbation theory:

$$
E(2)=-2 \frac{\left\langle n_{B}|F| \sigma_{X H}^{*}\right\rangle^{2}}{\varepsilon\left(\sigma_{X H}^{*}\right)-\varepsilon\left(n_{B}\right)}
$$

where $\left\langle n_{B}|F| \sigma_{X H}^{*}\right\rangle$ is the Fock matrix element between the $n_{B}$ and $\sigma_{X H}^{*}$ orbitals, $\varepsilon\left(\sigma_{X H}^{*}\right)-\varepsilon\left(n_{B}\right)$ is the orbital energy difference (the difference of diagonal Fock matrix element). It is worth mentioning that the CT and the corresponding lowering of energy are attributed to hydrogen bonding interactions. In other words, the second-perturbation energies $\mathrm{E}(2)$ lowering is responsible for the orbital interaction of $\mathrm{H}$-bond, the larger $\mathrm{E}(2)$ values correspond to stronger $\mathrm{CT}$ interaction occurring in the $\mathrm{H}$-bond. The results of $\mathrm{NBO}$ analysis are listed in table 3 .

In the NBO analysis of hydrogen bond system, the charge transfer between the lone pairs of proton acceptor and antibonds of the proton donor is the most important parameter. The results of NBO analysis illustrate that in the structure of Droxidopa, two lone pairs of oxygen atom $\left(\mathrm{n}_{2} \mathrm{O}\right)$ and a lone pair of nitrogen atom $(\mathrm{n} \mathrm{N})$ participate as donors and the $\sigma^{*}{ }_{\mathrm{O}-\mathrm{H}}$ interactions behave as acceptors in relatively strong intramolecular charge transfer interactions with the energy values presented in table 3 . The energy corresponds to the $\mathrm{n}_{2} \mathrm{O}(\mathrm{nN}) \rightarrow \sigma^{*}{ }_{\mathrm{O}-\mathrm{H}}$ interaction, which is connected with the maximum $\mathrm{n}_{2} \mathrm{O}(\mathrm{nN}) \rightarrow \sigma^{*}{ }_{\mathrm{O}-\mathrm{H}}$ overlap, leading to a linear, or nearly so, geometry of the O-H...O and $\mathrm{N}-\mathrm{H}$...O hydrogen-bonded system, respectively. Hydrogen bonding causes an increase of the occupancy of the $\sigma^{*}{ }_{\mathrm{O}-\mathrm{H}}$ antibond orbital and further weakening and lengthening of the $\mathrm{O}-\mathrm{H}$ bond. These orders of energy values again support the calculated intramolecular hydrogen bonds strength values in Droxidopa in $\mathrm{VAC}$ and in the five solvents.

It is worth mentioning that the NBO energy connected with $\mathrm{n}_{2} \mathrm{O}(\mathrm{nN}) \rightarrow \sigma^{*}{ }_{\mathrm{O}-\mathrm{H}}$ overlap nicely correlating with other geometrical and topological parameters. Moreover, we found out that there is an excellent

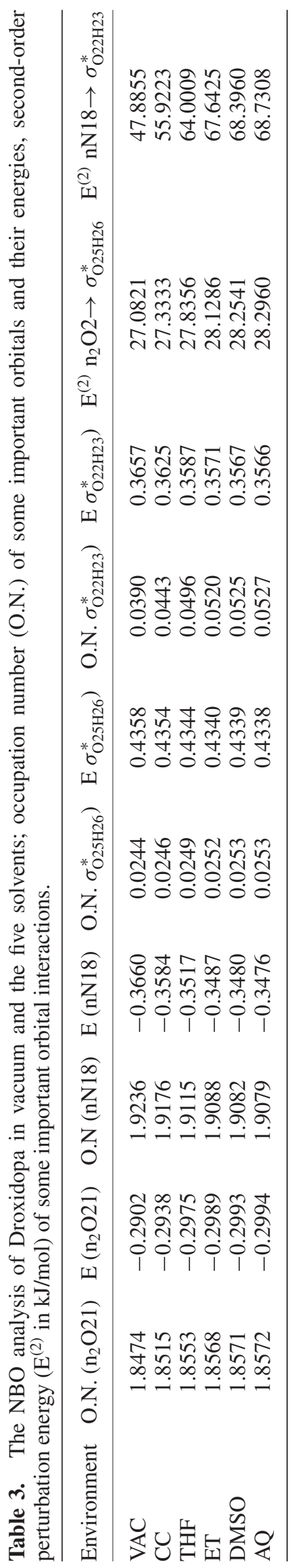


correlation between $\mathrm{E}_{\mathrm{HB}}$ versus $\mathrm{n}_{2} \mathrm{O} \rightarrow \sigma^{*}{ }_{\mathrm{O}-\mathrm{H}}$ and $\mathrm{nN} \rightarrow \sigma^{*}{ }_{\mathrm{O}-\mathrm{H}}$ with correlation coefficient of 0.955 and 1.00 , respectively. These kinds of correlations are important, because they permit us to quantitatively dictate the strength of such interactions and provide a physical explanation for the process. Eventually, the NBO and AIM analyses show that the strength of the IHB increases with the increase of the solvent dielectric constant.

\section{Conclusion}

We have examined two types of IHBs for Droxidopa in vacuum and five solvents using B3LYP level of theory at the $6311++\mathrm{G}(\mathrm{d}, \mathrm{p})$ basis set. The results obtained from the DFT calculations and the topological parameters derived from the Bader theory suggest that the stronger $\mathrm{HB}$ can lead to lengthening the $\mathrm{O}-\mathrm{H}$ bond and shortening the $\mathrm{N}$...H and the O...H distances. Our calculated RCP values are useful descriptors of the strength of IHBs, emphasizing that there is a correlation between $\mathrm{E}_{\mathrm{HB}}$ and the other parameters such as solvent dielectric constant. Moreover, the calculated electron densities and Laplacian properties of Droxidopa in vacuum and five solvents illustrate that $\mathrm{O}(\mathrm{N}) \ldots \mathrm{H}$ bonding have lower $\rho$ and positive $\nabla^{2} \rho$ values. These properties are typical for closed-shell interactions as HBs that indicate electrostatic characters of the $\mathrm{O}(\mathrm{N})$... H bondings. NBO analysis indicates that there is an excellent linear correlation between $\mathrm{E}_{\mathrm{HB}}$ and $\mathrm{n}_{2} \mathrm{O}(\mathrm{nN}) \rightarrow \sigma^{*}{ }_{\mathrm{O}-\mathrm{H}}$ charge transfer in vacuum and five solvents. Overall, our theoretical calculations show that the strength of the IHB increases with the increase of the solvent dielectric constant and we found a good correlation between these parameters.

\section{References}

1. Raissi H, Yoosefian M and Khoshkhou S 2012 Comput. Theor. Chem. 9831

2. Raissi H, Yoosefian M, Zamani S and Farzad F $2012 \mathrm{~J}$. Sulfur Chem. 3375

3. Raissi H, Yoosefian M, Mollania F, Farzad F and Nowroozi A R 2011 Comput. Theor. Chem. 966299

4. Raissi H, Yoosefian M and Mollania F 2012 Int. J. Quantum Chem. 1122782

5. Dunitz J D, In X-ray Analysis and the Structure of Organic Molecules (London: Cornell University Press) (1979)

6. Nadim E S, Raissi H, Yoosefian M, Farzad F and Nowroozi A R 2010 J. Sulfur Chem. 31275

7. Raissi H, Khanmohammadi A, Yoosefian $M$ and Mollania F 2013 Struct. Chem. 241121
8. Raissi H, Yoosefian M, Mollania F and Khoshkhou S 2013 Struct. Chem. 24123

9. Yoosefian M, Raissi H, Davamdar E, Esmaeili A and Azaroon M 2012 Chin. J. Chem. 30779

10. Yoosefian M, Raissi H, Nadim E S, Farzad F, Fazli M, Karimzade E and Nowroozi A 2011 Int. J. Quantum Chem. 1113505

11. Raissi H, Jalbout A, Nasseria M, Yoosefian M, Ghiassi H and Hameed A 2008 Int. J. Quantum Chem. 1081444

12. Raissi H, Yoosefian M, Hajizadeh A, Karimi M and Farzad F 2012 Bull. Chem. Soc. Jpn. 8587

13. Raissi H, Nadim E S, Yoosefian M, Farzad F, Ghiamati E, Nowroozi A R, Fazli M and Amoozadeh A $2010 \mathrm{~J}$. Mol. Struct. Theochem. 9601

14. Meng-Xia X and Yuan L 2002 Spectrochim. Acta, Part A 582817

15. Schlücker S, Singh R K, Asthana B, Popp J and Kiefer W 2001 J. Phys. Chem. A 1059983

16. Bonaccorsi R, Palla P and Tomasi J 1984 J. Am. Chem. Soc. 1061945

17. Del Valle F and Tomasi J 1987 Chem. Phys. 114231

18. Mammino L and Kabanda M M 2009 J. Phys. Chem. A 11315064

19. Alagona G, Ghio C, Igual J and Tomasi J $1990 \mathrm{~J}$. Mol. Struct. Theochem 204253

20. Barone V, Cossi M and Tomasi J 1997 J. Chem. Phys. 1073210

21. Cossi M, Scalmani G, Rega N and Barone V $2002 \mathrm{~J}$. Chem. Phys. 11743

22. Holmquist M, Hæffner F, Norin T and Hult K 1996 Protein Sci. 583

23. Bonaccorsi R, Hodošček M and Tomasi J $1988 \mathrm{~J}$. Mol. Struct. THEOCHEM. 164105

24. Nogrady T and Weaver D F 2005 In Medicinal Chemistry: A molecular and biochemical approach (New York: Oxford University Press)

25. Goldstein D S 2006 Cardiovasc. Drug Rev. 24189

26. Mathias C J 2008 Clin. Auton. Res. 1825

27. Tomasi J and Persico M 1994 Chem. Rev. 942027

28. Tomasi J, Mennucci B and Cammi R 2005 Chem. Rev. 1052999

29. Frisch M, Trucks G, Schlegel H, Scuseria G, Robb M, Cheeseman J, Montgomery Jr. J, Vreven T, Kudin K and Burant J, Gaussian 03, Rev B. 03. (Gaussian Inc, Pittsburgh, PA) (2003)

30. Biegler-Konig F 1998 In AIM 2000 (Germany: University of Applied Science)

31. Glendening E, Reed A, Carpenter J and Weinhold F, NBO, version 3.1. (Pittsburgh PA) (1988)

32. Schuster P, Zundel G and Sandorfy C (Eds.) 1976 Hydrogen bond; recent developments in theory and experiments (Amsterdam: North-Holland Publishing Company)

33. Espinosa E, Molins E and Lecomte C 1998 Chem. Phys. Lett. 285170

34. Abramov Y A 1997 Acta Crystallogr. Sect. A 53264

35. Bader R F 1990 In Atoms in Molecules: A Quantum Theory (Oxford: Oxford University Press)

36. Popelier P, Aicken F and O'Brien S 2000 Chemical Modelling: Applications and Theory 1143 